\title{
Product Review Based on Machine Learning Algorithms
}

\author{
D.Elangovan ${ }^{\mathrm{a}, 1}$, V.Subedha ${ }^{\mathrm{b}}$ \\ ${ }^{a, 1}$ Research Scholar, Sathyabama Institute of Science And Technology, Chennai \\ ${ }^{b}$ Professor \& Head, Panimalar Institute of Technology, Chennai
}

\begin{abstract}
The purchase of products or administrations through an electronic trade called web based shopping over internet using a web browser. Online Product surveys are significant for up and coming purchasers in helping them decide. To this end, distinctive sentiment mining systems have been proposed, where making a decision about a survey sentence's direction (e.g., positive or negative) is one of their key difficulties. As of late, Machine learning has risen as powerful methods for taking care of assumption order issues. An AI model inherently learns a helpful portrayal consequently without human endeavors. In any case, we propose a regulated AI structure for item audit conclusion arrangement which utilizes pervasively accessible evaluations as powerless supervision signals. To assess the proposed system, we build a dataset containing 2, 00,000 pitifully named survey sentences and 15000 marked audit sentences from Amazon. Trial results show the more exactness contrasted with past one.
\end{abstract}

Keywords - Machine learning, Sentimental analysis, opinion mining, sentimental classification.

\section{INTRODUCTION}

Machine learning is an exhibition paradigm utilizing model information or past experience.Machine Learning is the study of calculations that improve their presentation, at some worst experience,optimizes an exhibition standard utilizing model information or history of previous experiences. The role Role of Statistics is to Infer from an example and Computer science is efficient calculations to Solve the enhancement issue.

In olden days, the purchasing of items was progressively found on getting item audit from peer group like adjacent neighbors, relatives and friends as items were bought straightforwardly from dealers. Individuals trusted relatives, and companions audit about item supportive. Be that as it may, with change in innovation, we saw advancement of E-trade industry with locales overwhelmed results from various brands made accessible to clients at the dash of a single tick. The accessibility of item based destinations with doorstep conveyance has made it helpful for clients to shop on the web. It gives one stop shop to all needs of clients. With such a great amount of progress

\footnotetext{
${ }^{1}$ D.Elangovan, Research Scholar, Sathyabama Institute of Science and Technology Chennai.
}

Email: elangovan.durai@yahoo.com 
in shopping design, we see dealers furnishing clients with input alternative about the item. Clients compose surveys from all parts of the world. There are thousands, a huge number of audits being composed. So an inquiry emerges on the most proficient method to get essential judgment about item without experiencing every one of them independently. A large number of audits are very long as well as troublesome for a potential buyer to survey and analyze them to settle on a final choice on whether the buyer should buy the item or not. More number of audits will make it cumbersome for item producers or manufacturers to keep a $\log$ of client assessments and the assumptions communicated on their items and administrations. In this way it progresses and becomes a need to create a synopsis of surveys. The rundown of audits is finished utilizing opinion examination.

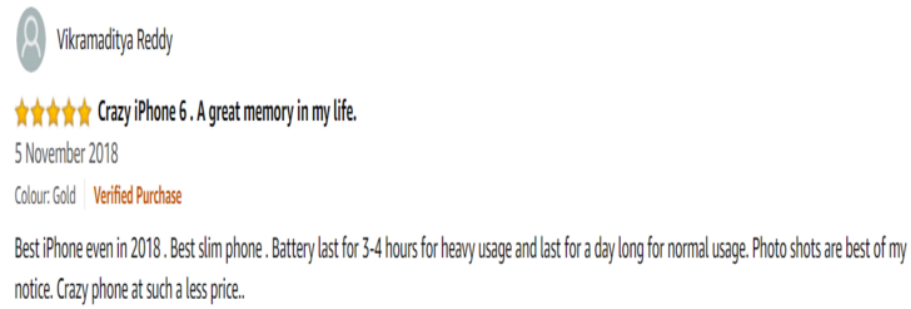

Figure 1. Positive Reviews

In general, Slant investigation concentrates more on emotional data required for source materials by applying normal idea of regular dialect handling the principle errand lies in distinguishing whether the assessment expressed is sure or negative. Since clients normally don't express feelings in basic way, here and there it winds up repetitive errand to pass judgment on a supposition expressed.

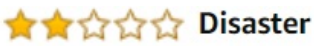 \\ 1 November 2018 \\ Colour: Gold $\quad$ Verified Purchase \\ When I trying to charge it,there's flowing currents in phone}

Figure 2. Negative Reviews

A few suppositions are proximal ones while others are immediate. The Nostalgic examination enables client to imagine fulfillment while obtaining the basic synopsis of these surveys classified into positive or negative-two more extensive arranged classes. After realizing current market drifts about items which is very useful for creating market methodologies by traders, the Criticisms are principally utilized by the buyers to purchase the goods and services on the web. 


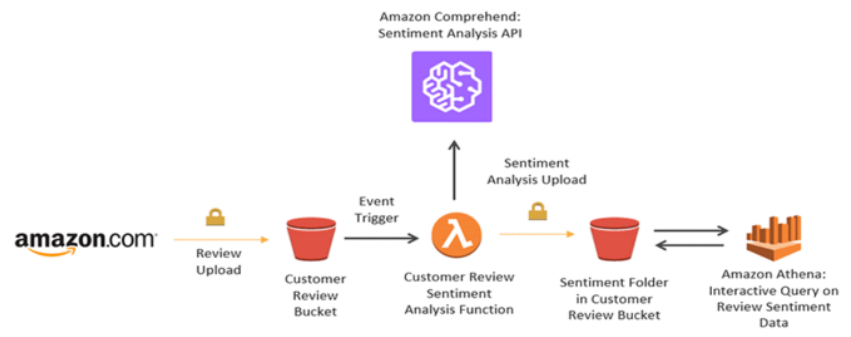

Figure 3. Overview of Amazon sentimental analysis

\section{RELATED WORKS}

Determining a consensus opinion on a product sold online is no longer simple as appraisals have become increasingly more on the Internet. To address this issue, analysts have utilized various like searching for sentiments communicated in the reports, investigating the appearance and sentence structure of audits. The Perspective based assessment is the most significant part of supposition mining and analysts are getting increasingly intrigued by item viewpoint extraction. The progressively complex calculations are expected to address this issue decisively with enormous informational indexes. This acquaints a strategy with extricate and abridge item angles and then compare sentiments from countless item audits in a particular area. This amplifies the exactness as well as convenience of the audit outlines utilizing information about item angle extraction and giving both a proper degree of detail and rich portrayal capacities.

Various researchers have worked in the field of end assessment, each one proposing better methodology for providing indications of progress adequacy from AI draws near. A LSA to separate thing incorporate inclination words which are required to get right sentences to wind a summary of reviews, with empowering just picked features to exhibit the last items, along these lines, reducing genuine size of once-over [1]. In [2] creator talks about the specific issues inside supposition assessment field which consolidates; report level, sentence level, feature level, comparative appraisal and conclusion jargon issue.

Bo hurt [4] considers masterminding reports not by subject, but instead overall inclination, wrapping up whether a study is positive or negative. The Overviews are changed over to essential decision by making use of systems. Since evaluations are not for each situation facilitate for instance "the nokia phone is extraordinary" yet moreover it might be a close to feeling like "nokia phone has best battery life over Samsung". There are three measurements namely sentence level, report level and feature level at which ends are gathered [8]. At sentence level, conceptual and target suppositions exist, whereas at document level, a record is portrayed reliant on all things considered presumption conveyed by end holder. At feature level, the characteristics of things are contemplated, which gives request through and through. In paper [5], an all encompassing vocabulary based methodology is suggested that enables the framework to deal with sentiment words that are setting subordinate. It considers the checking of 
the quantity of positive and negative supposition words close to the item include in each sentence. On the off chance that the number of positive feeling words are more than that of negative supposition words, the last expectation on the component is sure else negative. Author in [6] makes utilization of higher n-gram demonstrate utilizing three classifiers. The first being dialect demonstrate which is a generative strategy that processes the likelihood of age of a word arrangement. The Passive-Aggressive calculations are second which comprises of a group of edge based web based learning calculations for parallel characterization. Third, to foresee the extremity of an audit. Aside from characterizing audits in two more extensive classes, there likewise exists a term extremity degree to quantify the quality of sentiments, as in is the conclusion firmly positive, somewhat positive, and exceptionally negative and so forth [8].

Author in [9] says result of notion esteem and happening recurrence gives estimation of suppositions. The approach figures the general notion of expressed stubborn content like client audits and scales them as a genuine score between - 1 and +1 , which would then be able to be effectively changed positive/negative grouping or into a size of 1-5 stars. Making applicant list utilizing POS labelling with evacuation of stop word prompts viewpoint distinguishing proof. The viewpoint having fewer than 5 remarks on it, is expelled from the competitor list.

In paper [10], the item audit is converted into a Vector of Feature Intensities (VFI). A VFI is a vector of $\mathrm{N}+1$ esteem, every one speaking to an alternate item highlight and alternate highlights. Snyder and Barzilay [11] tended to the issue of investigating various related suppositions in a content and introduced a calculation that mutually gets the hang of positioning models for individual angles by demonstrating the conditions between allocated positions [20].

\section{PROPOSED SYSTEM}

\section{Dataset}

The dataset was gathered from various item destinations identified with portable space item audits like .cnet.com, downlaod.com, reviewcentre.com, zdnet.com, epinions.com and consumereview.com, Amazon.com

\section{Pre-Processing}

The unstructured dataset may contain dull words, large number of words that are in no way required in delineating of opinions. Pre-getting ready incorporates clearing of stop words, for instance, 'as well as', 'that, etc sought after by guard stemming which incorporates streamlining objective words into base words by ejection of increments like ed, ate, particle, ional, ment, ator, ssess, es, ance or transformation from ator to ate . For instance, "substitution" is stemmed to replace, "upset" to inconvenience, "glad" to happi and "administer" to work. The crude information is pre-handled to the show remarkable signs of quality improvement . 


\section{Extraction}

The features in surveys are extracted with the goal that it encourages the client to realize the positive and negative remarks of various features. Since, by and large the decision about item is truly necessary yet there is additionally circumstance where client prerequisites come into the situation. The utilization of descriptors is done using unigram model to order suppositions like positive or negative. For instance, "the Samsung camera I purchased was acceptable; it has got incredible touch screen, wonderful spotlight." The component separated out of it would resemble:Domain: Flexible; Product: Samsung; Feature: Camera; Adjective: Good.

\section{Compare Model}

Managed learning creates a capacity which maps contributions to wanted yields likewise called as marks since they are preparing models named by human specialists. The Naive-Bayes and Support Vector Machine methods are applied to complete regulated learning on the available datasets .

\section{The pseudo code}

Input- Dataset of Amazon product reviews

Output- positive and negative Classification

1: Preprocess the data

Removal of special characters

Removal of stop words

Stemming the word

2: feature list

If word in stop word list

Removal word

Return file

Else append word to file

3: Extract feature list

Match every word in pre processed list

If word matches adjective in base list

Display word

If word matches feature in base list

Display feature

4: combine both feature and preprocessed list

5: Use machine learning algorithms

Compute probability

6: classify opinion as positive, negative or neutral. 


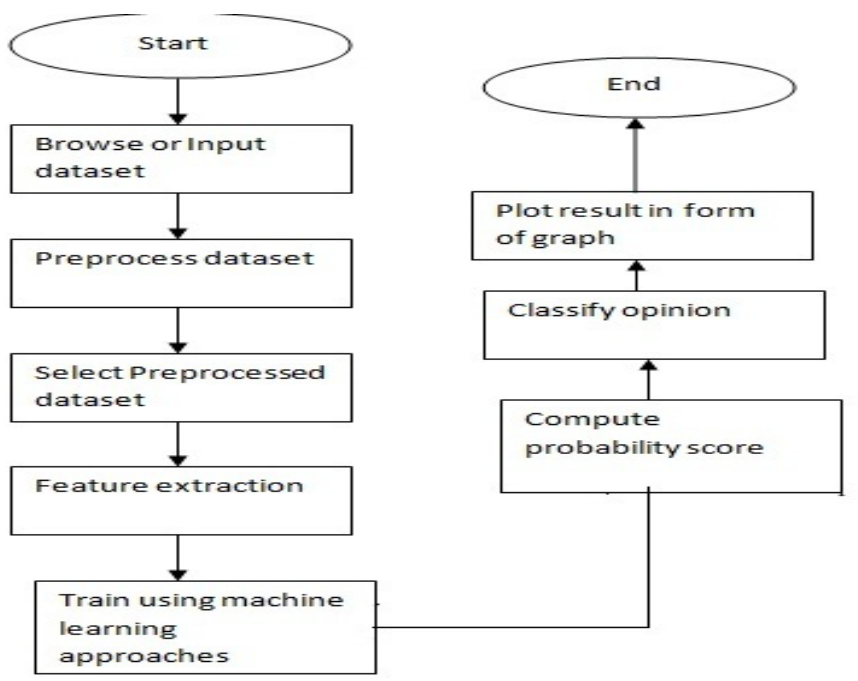

\section{CONCLUSION}

The Sentiment analysis is used to identify and aggregate the sentiment or opinions expressed by the users. It is used to classify the polarity of text in document or sentence in terms of the opinion expressed as positive, negative or neutral. Our proposed system is found to give better accuracy compared to previous approaches. It takes much more computation time for text files that are too large in size. The automatic sentimental analysis is very useful to identify and predict current and future trends.

The future scope of improvement is to

- Review the product based on opinion in different languages.

- Trade with problem of mapping slangs.

- Add sarcastic opinion.

- Recognizing similar feelings and discovering which among two item looked at is best one.

\section{REFERENCES}

[1] Liu, Chien-Liang, et al., Movie rating and review summarization in mobile environment. Systems, Man, and Cybernetics, Part C: IEEE Transactions on Applications and Reviews, Volume 42 issue 3, pp.397-407,2012.

[2] Feldman, Ronen. Techniques and applications for sentiment analysis.Communications of the ACM 5,Volume 56 Issue4, pp.82-89,2013. 
[3] Hearst, Marti A., Susan T. Dumais, Edgar Osman, John Platt, and Bernhard Scholkopf. Support vector machines. Intelligent Systems and their Applications, IEEE 13,Volume. 4 ,pp.18-28, 1998.

[4] Pang, Bo, , et al., Thumbs up?: sentiment classification using machine learning techniques. In Proceedings of the ACL-02 conference on Empirical methods in natural

[5] Ding, Xiaowen, et al., A holistic lexicon-based approach to opinion mining. Proceedings of the 2008 International Conference on Web Search and Data Mining. ACM, pp. 231-240, 2008.

[6] Cui, Hang, , et al., Comparative experiments on sentiment classification for online product reviews. Proceedings of the 21st national conference on Artificial intelligence (AAAI). Volume 2, pp 1265$1270,2006$.

[7] Jebaseeli, A, et al.,. A Survey on Sentiment Analysis of (Product) Reviews. International Journal of Computer Applications 47, Volume 11, pp36-39, 2012.

[8] Wang, Min, , et al., Research on sentiment analysis technology and polarity computation of sentiment words. Progress in Informatics and Computing (PIC), 2010 IEEE International Conference on. Vol. 1. IEEE, 2010

[9] Mudinas, Andrius, , et al., Combining lexicon and learning based approaches for concept-level sentiment analysis. Proceedings of the First International Workshop on Issues of Sentiment Discovery and Opinion Mining. ACM, pp330-333, 2012.

[10] de Albornoz, , et al. A joint model of feature mining and sentiment analysis for product review rating. Advances in information retrieval. Springer Berlin Heidelberg, pp.55-66, 2011.

[11] Mattosinho, F. , et al., Thesis on Mining Product Opinions and Reviews on the Web. Technische Universitat Dresden ,2010.

[12] Pang, Bo, et al., Thesis on Opinion mining and sentiment analysis. Foundations and trends in information retrieval Volume1-2,pp 1-135,2008

[13] Zhu, Jingbo, et al. Aspect-based opinion polling from customer reviews. IEEE Transactions on Affective Computing, Volume 2.1,pp.37-49, 2011.

[14] Na, Jin-Cheon, , et al., Effectiveness of simple linguistic processing in automatic sentiment classification of product reviews. Advances in Knowledge Organization Volume9, pp. 49-54, 2004.

[15] Nasukawa, Tetsuya, , et al., Sentiment analysis: Capturing favorability using natural language processing. In Proceedings of the 2nd international conference on Knowledge capture, ACM, pp. 7077, 2003.

[16] Li, Shoushan, Zhongqing Wang, , et al., Sentiment Classification with Polarity Shifting Detection. In Asian Language Processing (IALP), 2013 International Conference on, pp. 129-132. IEEE, 2013.

[17] M.Karamibekr,A. , et al., Oriented Sentiment Classification, Processed of the IEEE/WIC/ACM International Conferences on Web Intelligence and Intelligent Agent Technology (WI-IAT), Vol (1): pp. 327-331, 2012.

[18] Melville, Prem, , et al., Sentiment analysis of blogs by combining lexical knowledge with text classification. InProceedings of the 15th ACM SIGKDD international conference on Knowledge discovery and data mining, pp. 1275-1284. ACM, 2009.

[19] Abbasi, Ahmed, sentiment analysis in multiple languages: Feature selection for opinion classification in Web forums.mACM Transactions on Information Systems (TOIS) Volume 26 issue 3, pp.1- 12, 2008.

[20] Ambeth Kumar.V.D (2017).Efficient Routing for Low Rate Wireless Network a Novel Approach. International Journal of Image Mining, Vol. 2, Nos. 3/4, 2017, 2017 